\title{
Estimativas de custos visando orientar a tomada de decisão na implantação de redes, coletores e elevatórias de esgoto
}

\section{Cost estimates to guide decision making in the implemention of networks, sewage collectors and sewage pumping stations}

\author{
Rodrigo Pinheiro Pacheco ${ }^{1}$, Carlos Eduardo Curi Gallego ${ }^{2}$, Cristóvão V. S. Fernandes ${ }^{3}$ e Miguel Mansur Aisse $^{4}$ \\ ${ }^{1}$ Engenheiro da Cobrape - Cia Brasileira de Projetos e Empreendimentos \\ rodrigopacheco@cobrape.com.br \\ ${ }^{2}$ Engenheiro da Cobrape - Cia Brasileira de Projetos e Empreendimentos \\ cadu@cobrape.com.br \\ ${ }^{3}$ Professor Associado do Departamento de Hidráulica e Saneamento (DHS) e do PPGERHA da UFPR \\ cris.dhs@ufpr.br \\ ${ }^{4}$ Professor Associado do Departamento de Hidráulica e Saneamento (DHS) e do PPGERHA da UFPR \\ miguel.dhs@ufpr.br
}

Recebido: 07/02/14 - Revisado: 08/07/14 - Aceito: 27/08/14

\begin{abstract}
RESUMO
Apesar do transporte dos esgotos ser um assunto recorrente no saneamento básico, ainda não se encontram com frequência, os custos atualizados para a implantação dos elementos deste sistema. Visando orientar os estudos de viabilidade e as politicas para o afastamento dos esgotos, o objetivo deste trabalho foi desenvolver métodos e apresentar estimativas de custos de implantação para a coleta e transporte dos esgotos. Foram atualizados custos de projetos para implantação dos sistemas de transporte. Também foram desenvolvidos pré-dimensionamentos e seus respectivos orçamentos. Para as redes coletoras de esgoto, os resultados foram obtidos através do pré-dimensionamento, considerando diferentes niveis de declividade de terreno, diâmetros da tubulação de 150 a 350 mm e diferentes tipos de solos e urbanização. Para os Coletores e Interceptores foram atualizados os custos de projetos para desenvolver equaçoes, com DNs que variam de 200 a 800 mm, dentre materiais de PVC ao Concreto Armado. Para as Estaçoses Elevatórias de Esgoto - EE Es foram obtidas estimativas de custos para faixas de diferentes alturas manométricas, com relação à vazão em L/s. Para as Linhas de Recalque - LR encontrou-se estimativas de custo em função do Diâmetro Nominal - DN com a utilização de diferentes materiais (PEAD, PVC DEFoFo, FoFo e RPVC/PRFV).
\end{abstract}

Palavras Chave: Coleta e transporte de esgotos. Coletores e interceptores. Custos de implantação. Estação elevatória de esgotos. Rede coletora de esgotos.

\begin{abstract}
Although transporting sewage is a recurring topic in basic sanitation, updated costs to implement the elements of this system are not often found. Aiming to guide studies on feasibility and policies to remove sewage, the purpose of this study was to develop methods and, present cost estimates to implement sewage collection and transport. The costs of projects to implement the transport systems were updated. Pre-sizing was also developed with the respective budgets. For the sewage collector networks, the results were obtained by pre-sizing considering different levels of terrain slope, diameters of the conduits from 150 to 350 $\mathrm{mm}$, and different types of soils and urbanization. For the Collectors and Interceptors, the project costs were updated to develop equations with NDs that vary from 200 to $800 \mathrm{~mm}$ in materials ranging from PVC to Reinforced Concrete. For the Sewage Pumping Stations (EEE-Estaçoes Elevatórias de Esgoto), estimates of costs were obtained for different ranges of manometric heights related to the flow in $L / s$. For the Sewage Discharge Lines (LR-Linhas de Recalque), cost estimates were found based on the Nominal Diameter-ND, using different materials (PEAD, PVC DEFoFo, FoFo and RPVC/PRFV).
\end{abstract}

Keywords: Sewage collection and transport. Collectors and interceptors. Implementation costs. Sewage pumping stations. Sewage collector network. 


\section{INTRODUÇAO}

Segundo os dados oficiais do Governo Federal, divulgados no último Diagnóstico dos Serviços de Água e Esgoto (MINISTÉRIO DAS CIDADES, 2014), a cobertura de esgotamento sanitário no Brasil é da ordem de 48,3\% da população total (urbana e rural) para os esgotos coletados e apenas 38,7\% da população total para os esgotos tratados. $\mathrm{O}$ índice brasileiro do esgoto coletado indica que menos da metade dos domicílios possuem um sistema de coleta e transporte de esgotos até o destino final.

Para o transporte do esgoto são considerados seus integrantes as redes coletoras de esgoto, os coletores troncos, os interceptores, as estações elevatórias, as linhas de recalque, as caixas de reuniões, os condutos forçados e os emissários.

No Brasil, ainda há uma carência de informações, no que diz respeito os custos de implantação de sistemas de esgotamento sanitário. Isto não significa que eles não existam, como atestam ANA (2008), Brudeki e Aisse (2007), Lucca, Samways e Aisse (2011), Salazar e Von Sperling (2011), incluindo os custos de tratamento.

Para custos de implantações dos Sistemas de Esgotamento Sanitário (SES), Brudeki e Aisse (2007) encontraram o custo total de investimento na ordem de $\mathrm{R} \$ 523,23$ por habitante para o Sistema de Esgotamento no Estado do Paraná. Entretanto, sabe-se que os custos per capita para um sistema de menor vazão de projetos pode se apresentar maior e que os custos de infraestrutura também podem variar de região para região, respeitando as particularidades locais.

Colossi (2002) apresentou equações que podem ser atualizadas para efeitos de comparação. Entre as equações desenvolvidas por Colossi (2002) a equação 1 pode ser utilizada para se determinar os custos de implantação de estação elevatória de esgoto.

$y=3,29 \times 10^{3} L^{0,330} X^{0,679}$

Onde,

y é o custo da estação em moeda americana - 2002;

L é a altura manométrica (em metros) de recalque; e

$\mathrm{X}$ é a vazão (L/s) da EEEs.

Outra equação desenvolvida por Colossi (2002) é a equação 2 para determinar os custos das linhas de recalque (ou emissários):

$y=10,152 \mathrm{~L}^{1,044} \mathrm{X}^{0,548}$

Onde,

y é o custo da linha de recalque em moeda americana

-2002 ;

L é a extensão da linha de recalque (em metros); e

$\mathrm{X}$ é a vazão $(\mathrm{L} / \mathrm{s})$ de projeto da linha de recalque.

$\mathrm{Na}$ elaboração do orçamento para as curvas de custos para o transporte de efluentes líquidos, a Cobrape-EngecorpsGeoambiente (2009) apresentaram os custos de assentamento de tubulação de esgoto por metro linear através da equação 3 , onde x é o $\mathrm{DN}$ do tubo em mm e y o resultado em $\mathrm{R} \$ / \mathrm{m}$ : $y=0,6248 x^{1,1274}$

A equação foi aplicada para diâmetros de $150 \mathrm{~mm}$ a $1500 \mathrm{~mm}$, empregando PVC e concreto armado. Considerando apenas obras civis, resultou a equação 4 onde x é o DN do tubo em $\mathrm{mm}$ e y o resultado em $\mathrm{R} \$ / \mathrm{m}$ :

$y=0,2644 x^{1,221}$

Salazar e von Sperling (2011) apresentaram faixas de valores e funções de custo unitário de implantação de redes coletoras e interceptores de sistemas de esgotamento dinâmico. Os custos foram obtidos a partir de orçamentos por empresas de consultoria, com predominância daquelas desenvolvidas no Estado de Minas Gerais. Os resultados dos custos médios foram expressos em custos unitários que variam de $\mathrm{R} \$ 86,14 / \mathrm{m}$ a $\mathrm{R} \$$ $147,05 / \mathrm{m}$ de acordo com percentuais de presença de rocha e de pavimentação. Os autores concluíram também que os custos dos serviços representam cerca de 70 a $85 \%$ dos custos totais de implantação, sobrepujando os custos dos materiais.

Lucca, Samways e Aisse (2011) estudaram custos de implantação de redes coletoras em cidades com pequena população (1400 a 5500 habitantes). Foram estudados quatro projetos executivos (municípios de Agudos do Sul, Bocaiúva do Sul, Mandirituba e Quitandinha) fornecidos pela Companhia de Saneamento do Paraná - SANEPAR. Para os custo da rede encontrou a faixa de $\mathrm{R} \$ 89,34 / \mathrm{m}$ a $\mathrm{R} \$ 111,90 / \mathrm{m}$. Para Coletores encontrou-se de $\mathrm{R} \$ 148,10 / \mathrm{m}$ a $\mathrm{R} \$ 277,42 / \mathrm{m}$.

Contudo, no Brasil não é comum a divulgação de informações de custos de obras de saneamento e menos ainda, a compilação destes dados para que representem os valores financeiros realizados (COBRAPE-ENGECORPS-GEOAMBIENTE, 2009). É necessário reunir uma base de dados sólida e suficiente para elaborar estimativas de custo para implantação de Sistemas de Esgotamento Sanitário, no contexto de planejamento de soluções integradas, visando soluções viáveis e sustentáveis.

O objetivo deste trabalho foi desenvolver método para a estimativa de custos de coleta e transporte de esgoto, fornecendo subsídio às tomadas de decisões e no auxílio ao desenvolvimento dos Estudos de Concepção.

A relevância deste estudo, além de dar subsídios às tomadas de decisões no setor de saneamento, também é utilizar as curvas para os custos de SES como uma ferramenta que possa auxiliar as tomadas de decisões na área do esgotamento sanitário, avaliando a melhor alternativa para implantação do sistema. Bem como, buscar uma padronização dos métodos para obter os custos das estruturas dos SES, minimizando as distorções e disparidades.

\section{MÉTODOS}

\section{Redes Coletoras de Esgoto}

$\mathrm{Na}$ obtenção dos custos de implantação da rede coletora de esgoto, observou-se que os fatores que mais influenciam são: (i) material, (ii) diâmetro, (iii) profundidade, (iv) extensão, (v) 
grau de urbanização e (vi) tipo do solo.

Neste caso as redes coletoras de esgoto foram orçadas, considerando algumas condições que podem ser encontradas in loco, fato que possibilitou desenvolver uma matriz de orçamentos, de acordo em que os parâmetros são alterados, conforme apresentado na sequência:

- Rede coletora com solo favorável (escavação mecanizada, solo com menos presença de rocha e distância pequena para transporte de bota-fora) e baixa urbanização (pouca presença de calçadas e asfaltos para recomposição e pouca interferência para execução da rede); - Rede coletora com solo desfavorável (escavação mecanizada e manual, solo com maior presença de rocha e distância grande para transporte de bota-fora) e baixa urbanização;

- Rede coletora com solo favorável e alta urbanização (maior presença de calçadas e asfaltos para recomposição e maior incidência de interferências na implantação da rede); e

- Rede coletora com solo desfavorável e alta urbanização. - Os dados de entrada para os custos das redes coletores de esgoto foram (a) área da sub-bacia, (b) população de saturação da sub-bacia e (c) nível de declividade do terreno.

Quanto ao (i) material para as redes, há vários tipos disponíveis no mercado. Os exemplos mais comuns de tubos para esgotamento sanitário são: cerâmicos, de PVC, de PRFV, de concreto armado, de FoFo, de FD, de aço galvanizado. Existe uma relação financeira entre o material e o diâmetro do tubo que depende do custo do metro linear do material. A partir do acréscimo do diâmetro o que se percebe é o uso de outros materiais considerados alternativos, tornando-se mais viáveis, além dos tubos de PVC e do concreto armado - mais utilizados atualmente.

Para as redes coletoras de esgoto foram considerados apenas o tubo PVC. Desta forma, este estudo considerou apenas o (ii) diâmetro do tubo que será determinado através do número de habitantes (dado de entrada), da área a ser esgotada, utilizando-se da Tabela 1 (COBRAPE-ENGECORPS-GEOAMBIENTE, 2009). Considerando os dados de projetos existentes, foi realizada uma distribuição dos diâmetros pela capacidade de escoamento por gravidade, obedecendo ao limite de vazão da tubulação descrita para a inclinação de $\mathrm{i}=0,04 \%$.

Quanto à (iii) profundidade, foi necessário classificar as redes em relação ao tipo de escoramento. As profundidades dos tubos estão diretamente correlacionadas com a declividade do terreno. A Tabela 2 apresenta os tipos de escoramentos comuns utilizados como parâmetro de projeto e obra, com suas respectivas faixas de profundidade.

Para determinar as profundidades, foram atribuídos percentuais de escoramento. Neste caso foram utilizadas como estudo de caso as informações contidas nas planilhas de dimensionamento das redes coletoras, sendo 70 sub-bacias do Projeto de SES Porto Velho (HAGAPLAN-COBRAPE, 2008). Estas informações foram organizadas, classificadas e parametrizadas (de acordo com os parâmetros da Tabela 2), conforme apresentado na Tabela 3.
Os cálculos das porcentagens por nível de terreno foram considerados e adotados os seguintes parâmetros:

- Porcentagens das extensões nas faixas de escoramentos para cada sub-bacia de acordo com a Tabela 3;

- Pesos para cada faixa de escoramento, proporcionais aos custos de implantação (preço Sabesp) da respectiva faixa de escoramento;

- Classificação das sub-bacias por ordem crescente em relação à soma dos pesos;

- Foram adotados cinco níveis para classificação crescente das sub-bacias da média aritmética dos escoramentos sendo, de 0 a 10\% para o nível 1, de 10 a 30\% para o nível 2, de 30 a $60 \%$ para o nível 3, de 60 a $90 \%$ para - o nível 4 e de 90 a $100 \%$ para o nível 5;

O (v) grau de urbanização também é um fator que pode ter influência direta nos custos das redes, ou seja, quanto mais urbanizada é área de implantação (ou sub-bacia), maior é a chance de encontrar interferências, tais como: redes de distribuição de água, redes de drenagem, redes elétrica e telefônica e travessias (de córregos, rodovias e ferrovias) entre outros casos particulares, além dos custos adicionais de recomposição de calçadas e asfalto.

Outro fator determinante é o (vi) tipo do solo onde as redes serão implantadas. Para diferentes tipos de solo existem diferentes tecnologias de execução que possuem influência direta nos custos. O resultado para as redes coletoras foram apresentados em $\mathrm{R} \$ / \mathrm{m}$, em quatro matrizes para cada situação de área de implantação, conforme comentado.

Ressalta-se que é importante estar atendo as restrições dos usos destes custos: o material orçado é o PVC, método construtivo das redes é à céu aberto e composição do orçamento é o padrão Sanepar - Companhia de Saneamento do Paraná.

\section{Coletores e Interceptores}

Para a obtenção das equações para os custos de coletores e interceptores, foram utilizados 55 orçamentos de projetos, atualizados através do Índice Nacional da Construção Civil INCC da Fundação Getúlio Vargas - FGV. Destes orçamentos 5 são do Estado de Espírito Santo, 9 do Paraná, 10 de Rondônia e 31 de São Paulo. Foram classificados por diâmetro, material e o resultado final para os custos dos coletores e interceptores apresentados em $\mathrm{R} \$ / \mathrm{m}$.

\section{Estações Elevatórias de Esgoto}

Inicialmente foram realizadas atualizações de 105 orçamentos de elevatórias de esgoto através do Índice Nacional da Construção Civil - INCC da Fundação Getúlio Vargas - FGV. Os orçamentos são 11 do Estado do Espírito Santo, 8 do Maranhão, 15 do Paraná, 23 de Rondônia e 48 de São Paulo.

$\mathrm{Na}$ obtenção dos custos de elevatórias de esgoto foi comum observar diferentes custos para mesma vazão de recalque, por influência dos seguintes fatores:

- Altura manométrica da linha de recalque;

- Tipo do conjunto bomba utilizado (submersível (96\%), autoescorvante $(2 \%)$, deslocamento positivo $(2 \%)$; e 
- Padrão construtivo da estrutura civil da EEE (profundidade do poço, tanque de acúmulo, guindaste, entrada, gerador de energia etc.).

- Como a maioria das EEE apresentadas (96\%) eram do tipo submersível, para este estudo, propõe-se a seguinte classificação, de acordo com a tabela 4 que é baseada apenas nas diferentes alturas manométricas.

- Então, os dados de entrada visando determinar o custo de implantação da EEE são: vazão (L/s) e altura manométrica (m.c.a.).

\section{Linhas de Recalque (Emissários)}

Inicialmente foram realizadas atualizações dos custos das linhas de recalque através do Índice Nacional da Construção
Civil - INCC da Fundação Getúlio Vargas - FGV. Foram utilizados 137 orçamentos, sendo 11 do Estado do Espírito Santo, 37 do Maranhão, 12 do Paraná, 24 de Rondônia e 43 de São Paulo.

Após a atualização dos custos das linhas de recalque, foi necessário classificá-las de acordo com os materiais, pois, percebeu-se uma grande dispersão dos valores dos materiais (PEAD, PVC, PVC DeFoFo, FoFo, FD, PRFV e RPVC) para o mesmo diâmetro nominal.

O dado de entrada da equação, para se determinar os custos das linhas de recalque é o DN, que na ausência deste dado, pode ser determinado através equação de Bresse, por exemplo.

Desta forma, a equação obtida pelo gráfico do tipo custo por vazão foi expressa em função DN e apresenta o resultado em $\mathrm{R} \$ / \mathrm{m}$.

Tabela 1 - Distribuição da composição dos diâmetros da rede de transporte de esgotos por faixas de população de saturação

\begin{tabular}{|c|c|c|c|c|c|c|c|}
\hline \multirow{2}{*}{ População (hab) } & \multicolumn{7}{|c|}{ Diâmetros Nominais (mm) } \\
\hline & 150 & 200 & 250 & 350 & 500 & 800 & 1000 \\
\hline $1-5.000$ & $100 \%$ & & & & & & \\
\hline $5.001-10.000$ & $80,00 \%$ & $20,00 \%$ & & & & & \\
\hline $10.001-20.000$ & $72,73 \%$ & $18,18 \%$ & $9,09 \%$ & & & & \\
\hline $20.001-50.000$ & $69,57 \%$ & $17,39 \%$ & $8,70 \%$ & $4,35 \%$ & & & \\
\hline $50.001-100.000$ & $68,09 \%$ & $17,02 \%$ & $8,51 \%$ & $4,26 \%$ & $2,13 \%$ & & \\
\hline $100.001-200.000$ & $67,37 \%$ & $16,84 \%$ & $8,42 \%$ & $4,21 \%$ & $2,11 \%$ & $1,05 \%$ & \\
\hline $200.001-500.000$ & $67,02 \%$ & $16,75 \%$ & $8,38 \%$ & $4,19 \%$ & $2,09 \%$ & $1,05 \%$ & $0,52 \%$ \\
\hline
\end{tabular}

Fonte: Cobrape-Engecorps-Geoambiente (2009)

Tabela 2 - Tipo de escoramento por profundidade de escavação

\begin{tabular}{c|c}
\hline Profundidade $(\mathrm{m})$ & Tipo de Escoramento \\
\hline Até 1,50 & Sem escoramento \\
\hline $1,50-1,70$ & Pontalete \\
\hline $1,70-2,30$ & Descontínuo \\
\hline $2,30-3,00$ & Contínuo \\
\hline $3,00-4,00$ & Especial \\
\hline $4,00-10,00$ & Metálico e Madeira \\
\hline
\end{tabular}

Tabela 3 - Distribuição da composição do tipo de escoramento por nível de declividade terreno

\begin{tabular}{|c|c|c|c|c|c|c|}
\hline $\begin{array}{c}\text { Nível de } \\
\text { declividade }\end{array}$ & $\begin{array}{c}\text { Sem } \\
\text { Escoramento }\end{array}$ & Pontalete & Descontínuo & Contínuo & Especial & $\begin{array}{c}\text { Metálico e } \\
\text { Madeira }\end{array}$ \\
\hline 1 & $81 \%$ & $5 \%$ & $11 \%$ & $3 \%$ & & \\
\hline 2 & $66 \%$ & $8 \%$ & $16 \%$ & $9 \%$ & $1 \%$ & $0 \%$ \\
\hline 3 & $57 \%$ & $7 \%$ & $16 \%$ & $14 \%$ & $5 \%$ & $1 \%$ \\
\hline 4 & $48 \%$ & $6 \%$ & $15 \%$ & $15 \%$ & $11 \%$ & $5 \%$ \\
\hline 5 & $23 \%$ & $3 \%$ & $18 \%$ & $21 \%$ & $20 \%$ & $15 \%$ \\
\hline
\end{tabular}

Tabela 4 - Classificação das alturas manométricas para conjuntos mombas das EEEs

\begin{tabular}{c|c}
\hline Altura Manométrica & Classificação \\
\hline Até 15 m.c.a. & Baixa \\
\hline De 15 a 30 m.c.a. & Média Baixa Alta \\
\hline De 30 a 45 m.c.a. & Alta
\end{tabular}




\section{RESULTADOS E DISCUSSÃO}

\section{Rede Coletora de Esgoto}

Os resultados dos custos das redes coletoras de esgoto, por diâmetro, de acordo com o nível do terreno (1 a 5), podem ser observados nas tabelas 5 a 8 .

Na tabela 5 apresentada é possível observar que o custo das Redes Coletoras de Esgoto (RCE) tem um acréscimo de até 46\% para o DN $150 \mathrm{~mm}$, de acordo com o nível de terreno aumenta. Para o DN $350 \mathrm{~mm}$ este acréscimo é de 31\%, considerando a mesma situação. Lembrando que o nível 1 possui declividades mais favoráveis ao transporte, e o nível 5 possui declividades menos favoráveis, com terrenos mais planos, o que resulta, consequentemente, num aprofundamento da rede.

$\mathrm{Na}$ tabela 6 apresentada observa-se as mesmas características, entretanto, a condição do solo é mais desfavorável. Para o pré-dimensionado desta tabela foi considerada a presença de $10 \%$ de rocha branda, maior distância da jazida para o transporte de solos e mais escavação e compactação manual, o que resultou numa diferença de aproximadamente 38\% para o DN $150 \mathrm{~mm}$ e $27 \%$ para o DN $350 \mathrm{~mm}$, em relação à tabela 5 .

Na tabela 7 apresenta-se um solo favorável, mas alta urbanização, com mais interferências e recomposição de pavimento maior, o que resultou numa diferença de aproximadamente 38\% para o DN $150 \mathrm{~mm}$ e 25\% para o DN $350 \mathrm{~mm}$, em relação à tabela 6 .

$\mathrm{Na}$ tabela 8 apresentam-se as condições mais extremas

Tabela 5 - Custo da RCE (R\$/m) para solo favorável e baixa urbanização

\begin{tabular}{|c|c|c|c|c|c|}
\hline Nível (1) & $\begin{array}{c}\text { DN } 150 \mathrm{~mm} \\
(\mathrm{R} \$ / \mathrm{m})\end{array}$ & $\begin{array}{c}\text { DN } 200 \mathrm{~mm} \\
(\mathrm{R} \$ / \mathrm{m})\end{array}$ & $\begin{array}{c}\text { DN } 250 \mathrm{~mm} \\
(\mathrm{R} \$ / \mathrm{m})\end{array}$ & $\begin{array}{l}\text { DN } 300 \mathrm{~mm} \\
\quad(\mathrm{R} \$ / \mathrm{m})\end{array}$ & $\begin{array}{l}\text { DN } 350 \mathrm{~mm} \\
\text { (R\$/m) }\end{array}$ \\
\hline 1 & 105,08 & 124,08 & 156,71 & 197,34 & 228,68 \\
\hline 2 & 124,04 & 143,40 & 176,37 & 217,36 & 249,04 \\
\hline 3 & 137,97 & 157,72 & 191,09 & 232,47 & 264,55 \\
\hline 4 & 150,97 & 171,35 & 205,36 & 247,37 & 280,08 \\
\hline 5 & 196,28 & 218,70 & 254,74 & 298,79 & 333,53 \\
\hline
\end{tabular}

NOTA: (1) Nível de declividade de terreno, ver tabela 3 .

Tabela 6 - Custo da RCE (R\$/m) para solo desfavorável e baixa urbanização

\begin{tabular}{|c|c|c|c|c|c|}
\hline Nível (1) & $\begin{array}{c}\text { DN } 150 \mathrm{~mm} \\
(\mathrm{R} \$ / \mathrm{m})\end{array}$ & $\begin{array}{c}\mathrm{DN} 200 \mathrm{~mm} \\
(\mathrm{R} \$ / \mathrm{m})\end{array}$ & $\begin{array}{c}\mathrm{DN} 250 \mathrm{~mm} \\
(\mathrm{R} \$ / \mathrm{m})\end{array}$ & $\begin{array}{c}\text { DN } 300 \mathrm{~mm} \\
(\mathrm{R} \$ / \mathrm{m})\end{array}$ & $\begin{array}{c}\text { DN } 350 \mathrm{~mm} \\
(\mathrm{R} \$ / \mathrm{m})\end{array}$ \\
\hline 1 & 129,68 & 150,75 & 185,44 & 228,15 & 261,56 \\
\hline 2 & 152,17 & 173,77 & 209,01 & 252,26 & 286,22 \\
\hline 3 & 170,53 & 192,77 & 228,65 & 272,54 & 307,14 \\
\hline 4 & 190,97 & 214,24 & 251,13 & 296,04 & 331,66 \\
\hline 5 & 256,89 & 283,08 & 322,90 & 370,73 & 409,28 \\
\hline
\end{tabular}

NOTA: (1) Nível de declividade de terreno, ver tabela 3.

Tabela 7 - Custo da RCE (R\$/m) para solo favorável e alta urbanização

\begin{tabular}{|c|c|c|c|c|c|}
\hline Nível ${ }^{(1)}$ & $\begin{array}{c}\text { DN } 150 \mathrm{~mm} \\
(\mathrm{R} \$ / \mathrm{m})\end{array}$ & $\begin{array}{c}\text { DN } 200 \mathrm{~mm} \\
(\mathrm{R} \$ / \mathrm{m})\end{array}$ & $\begin{array}{c}\text { DN } 250 \mathrm{~mm} \\
(\mathrm{R} \$ / \mathrm{m})\end{array}$ & $\begin{array}{c}\text { DN300 mm } \\
(\mathrm{R} \$ / \mathrm{m})\end{array}$ & $\begin{array}{c}\text { DN } 350 \mathrm{~mm} \\
(\mathrm{R} \$ / \mathrm{m})\end{array}$ \\
\hline 1 & 134,85 & 155,69 & 190,17 & 232,67 & 265,91 \\
\hline 2 & 154,76 & 175,96 & 210,80 & 253,66 & 287,25 \\
\hline 3 & 169,77 & 191,37 & 226,61 & 269,88 & 303,87 \\
\hline 4 & 184,04 & 206,28 & 242,17 & 286,08 & 320,71 \\
\hline 5 & 232,95 & 257,25 & 295,20 & 341,18 & 377,88 \\
\hline
\end{tabular}

Tabela 8 - Custo da RCE (R\$/m) para solo desfavorável e alta urbanização

\begin{tabular}{|c|c|c|c|c|c|}
\hline Nível (1) & $\begin{array}{c}\text { DN } 150 \mathrm{~mm} \\
(\mathrm{R} \$ / \mathrm{m})\end{array}$ & $\begin{array}{c}\text { DN } 200 \mathrm{~mm} \\
(\mathrm{R} \$ / \mathrm{m})\end{array}$ & $\begin{array}{c}\text { DN } 250 \mathrm{~mm} \\
(\mathrm{R} \$ / \mathrm{m})\end{array}$ & $\begin{array}{c}\text { DN 300 mm } \\
(\mathrm{R} \$ / \mathrm{m})\end{array}$ & $\begin{array}{c}\mathrm{DN} 350 \mathrm{~mm} \\
(\mathrm{R} \$ / \mathrm{m})\end{array}$ \\
\hline 1 & 166,67 & 190,45 & 227,94 & 273,53 & 309,91 \\
\hline 2 & 190,68 & 215,05 & 253,13 & 299,31 & 336,29 \\
\hline 3 & 210,87 & 235,94 & 274,72 & 321,60 & 359,28 \\
\hline 4 & 233,90 & 260,10 & 300,01 & 348,01 & 386,81 \\
\hline 5 & 307,03 & 336,39 & 379,47 & 430,65 & 472,62 \\
\hline
\end{tabular}

NOTA: ${ }^{(1)}$ Nível de declividade de terreno, ver tabela 3. 
com o solo favorável e alta urbanização. O que resultou numa diferença de aproximadamente 51\% para o DN $150 \mathrm{~mm}$ e $37 \%$ para o DN 350 mm, em relação à tabela 1.

Para se determinar o custo final da rede de uma área é necessário utilizar-se de taxas de implantação de rede, como por exemplo, de 180 a $240 \mathrm{~m} /$ ha, conforme o sistema viário.

\section{Coletores e Interceptores}

A seguir estão apresentados os resultados dos custos dos Coletores e Interceptores por faixa de diâmetro nominal - DN. O dado de entrada das equações de potência está em função de "x", diâmetro nominal - DN (em mm) do tubo.

Para classificar os diferentes DNs no mesmo coletor e interceptor foi escolhido um DN', de acordo com melhor relação $\mathrm{R} \$ / \mathrm{m}$. A figura 1 apresenta a curva e equação para os custos de coletores e interceptores de DN 200 a 400 mm.

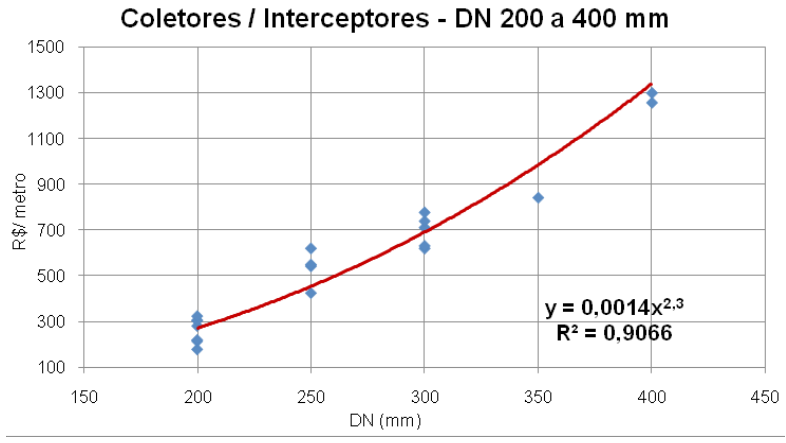

Figura 1 - Custo dos Coletores/ Interceptores DN 200 a $400 \mathrm{~mm}$

A figura 2 ilustra os custos de coletores e interceptores para $\mathrm{DN}$ de 400 a $800 \mathrm{~mm}$.

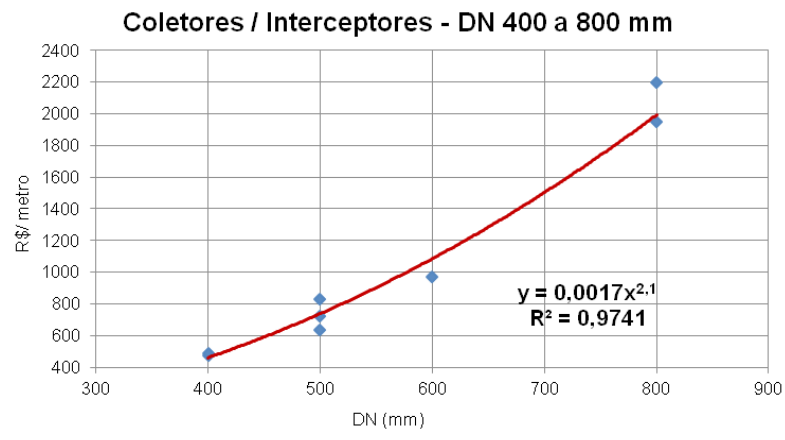

Figura 2 - Custo dos Coletores/ Interceptores DN 400 a $800 \mathrm{~mm}$

Para se determinar o custo final destas obras lineares é necessário estimar a sua extensão e DN, de acordo com o estudo de concepção ou alternativas de projeto a ser avaliadas. Outra opção é utilizar-se das relações de DN e população da tabela 1, sendo um pré-requisito conhecer a população correspondente do esgoto a ser transportado.

\section{Estações Elevatórias de Esgoto}

A seguir (Figuras 3 a 6) estão apresentados os resultados para as estações elevatórias de esgoto - EEEs, considerando as diferentes classificações das alturas manométricas, sendo a vazão (em L/s) adotada como a variável " $\mathrm{x}$ " da equação.

As estações elevatórias de esgoto nesta faixa de altura manométrica não possuem grande vazão, variando de 2,64 L/s a $39,87 \mathrm{~L} / \mathrm{s}$, como é apresentado na figura 3.

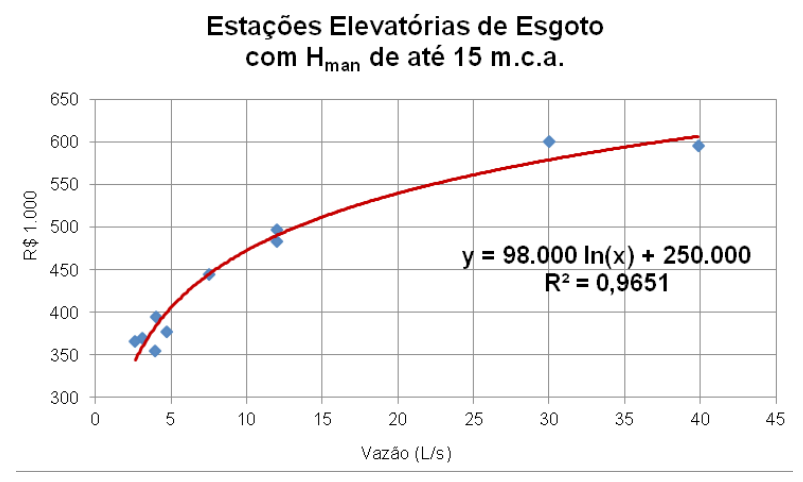

Figura 3 - Custo de EEE com Hman de até 15 m.c.a

A linha de tendência que melhor representou os custos das estações elevatórias de esgoto $H_{\operatorname{man}}$ até 15 m.c.a. foi a logarítmica, pois, foi observado que mesmo para elevatórias pequenas é necessário uma estrutura civil mínima, mas com o acréscimo de vazão este custo vai se diluindo com o ganho em escala.

As estações elevatórias de esgoto na faixa de altura manométrica de 15 até 30 m.c.a possuem uma vazão maior, variando de $3,10 \mathrm{~L} / \mathrm{s}$ a $380,00 \mathrm{~L} / \mathrm{s}$, como é apresentado na figura 4.

A linha de tendência que melhor representou os custos das estações elevatórias de esgoto $H_{\operatorname{man}}$ até 15 a 30 m.c.a. também foi a logarítmica. O acréscimo de vazão faz o custo inicial da unidade pode ser diluído com o ganho em escala.

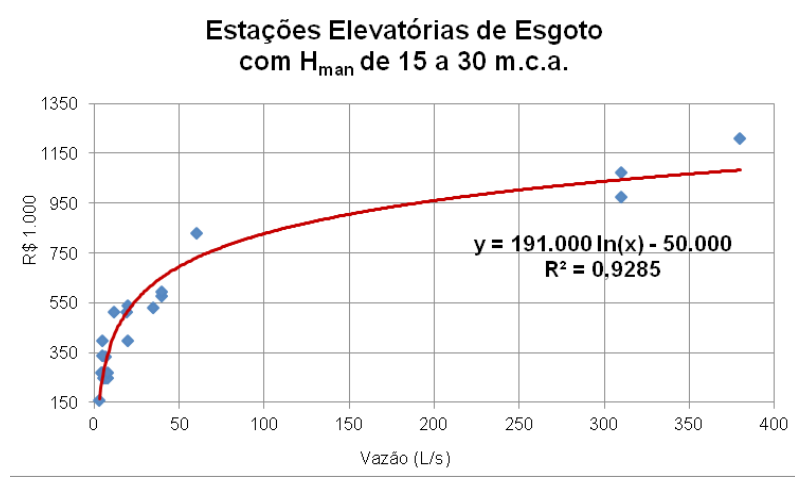

Figura 4 - Custo de EEE com $\mathrm{H}_{\text {man }}$ de 15 a 30 m.c.a

As estações elevatórias de esgoto na faixa de altura manométrica de 30 até 45 m.c.a possuem uma vazão intermediária de até $200,00 \mathrm{~L} / \mathrm{s}$, como é apresentado na figura 5 .

$\mathrm{Na}$ figura 5 também é possível observar que a linha de tendência escolhida para representar os custos das estações elevatórias de esgoto $\mathrm{H}_{\operatorname{man}}$ até 30 a 45 m.c.a. foi a logarítmica. 


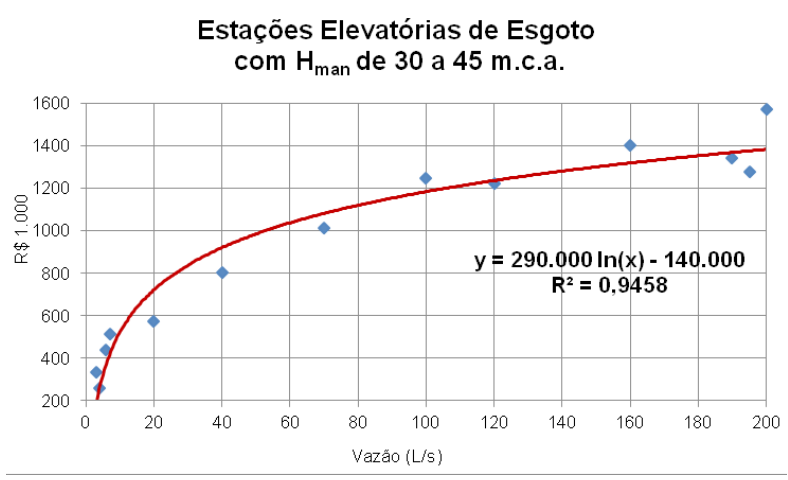

Figura 5 - Custo de EEE com hman de 30 a 45 m.c.a.

Percebe-se que as estações elevatórias de esgoto na faixa de altura manométrica acima de 45 m.c.a possuem vazões maiores, que variam de aproximadamente 5 a 260,00 L/s. A equação escolhida para representar os custos das EEEs com $\mathrm{H}_{\operatorname{man}}$ acima de 45 m.c.a. é a logarítmica, com um $\mathrm{R}^{2}$ de 0,9274 , que mostra uma boa relação entre os pontos e curva.

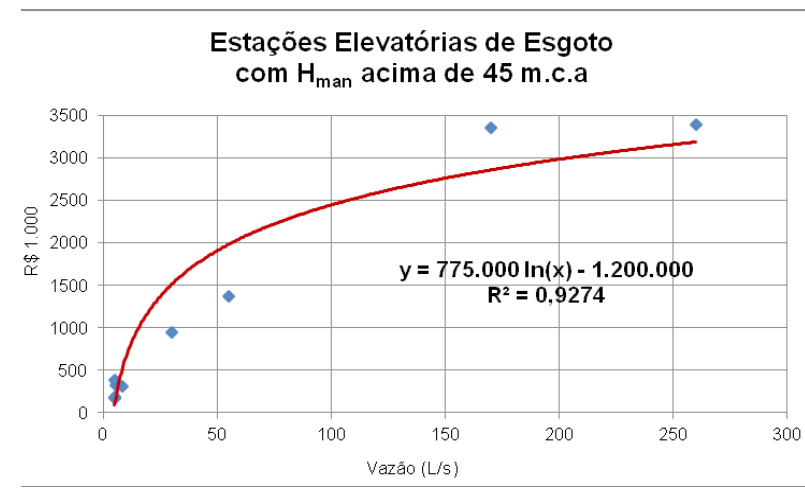

Figura 6 - Custo de EEE com Hman acima de 45 m.c.a Linhas de Recalque

A seguir (Figuras 7 a 11) estão apresentados os resultados dos custos para as linhas de recalque, também denominadas como emissários.

A equação que melhor pode exprimir os custos para as linhas de recalque para o material PEAD foi a equação do tipo potência, figura 7. A entrada da equação é dada em função de "x" que é o diâmetro nominal - DN (em mm) da linha de recalque.

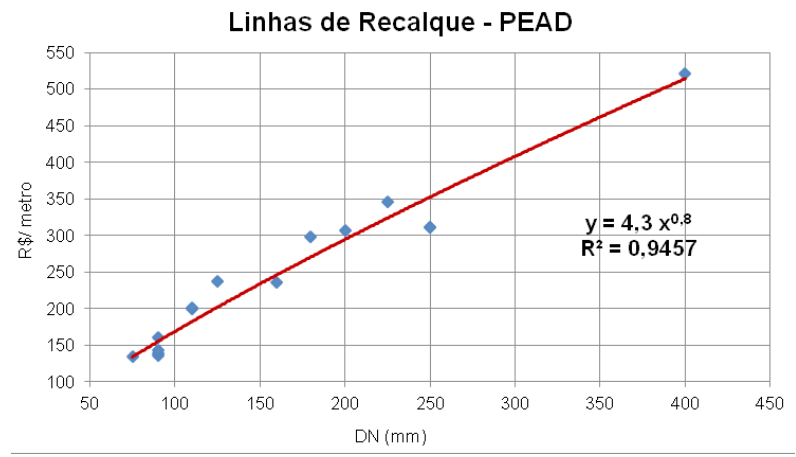

Figura 7 - Custo de Linhas de Recalque - PEAD
Para as linhas de recalque de PVC DeFoFo também se observou um acréscimo no valor $\mathrm{R} \$ / \mathrm{m}$ conforme o diâmetro aumenta, porém este valor mostrou-se mais econômico em relação ao PEAD.

Entretanto, os transientes hidráulicos da linha que recalque também podem influenciar na escolha do tubo, pois, é necessário que o material escolhido suporte as pressões positivas e negativas da linha.

A equação escolhida para representar os custos das linhas de recalque de material PVC e DeFoFo (figura 8) foi a do tipo potência. A entrada da equação é dada em função de "x" que é o diâmetro nominal - DN da linha de recalque.

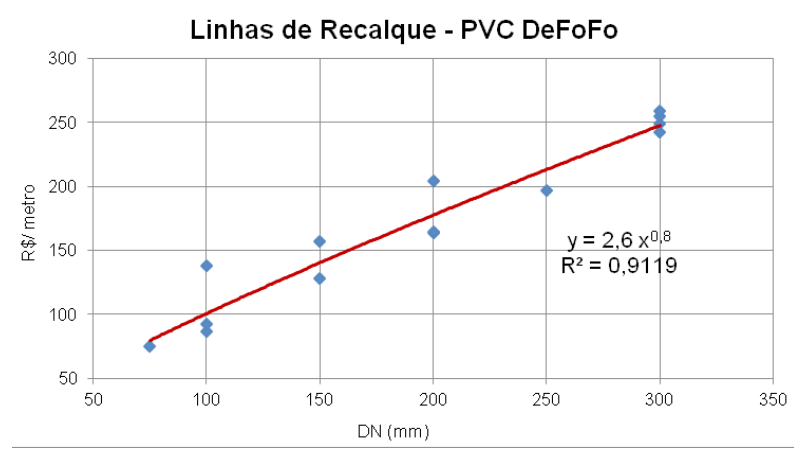

Figura 8 - Custo de Linhas de Recalque - PVC DeFoFo

A equação escolhida para representar os custos da figura 9 (linha de recalque em FoFo) foi a logarítmica, pois, conforme o DN aumenta observou-se uma leve acentuação dos custos. A entrada da equação é dada em função de " $x$ " que é o diâmetro nominal - DN (em mm) da linha de recalque.

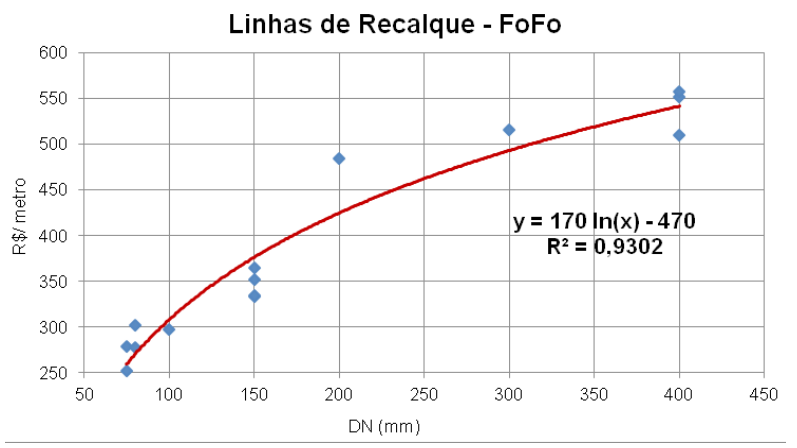

Figura 9- Custo de Linhas de Recalque - FoFo

Neste caso, como se observa na figura 10, foi adotada a equação linear para representar os custos das linhas de recalque - PRFV / RPVC. A entrada da equação é dada em função de " $\mathrm{x}$ " que é o diâmetro nominal $-\mathrm{DN}$ da linha de recalque.

Para se obter o custo final da linha é necessário conhecer a extensão total. Neste caso, para as extensões, pode se utilizar os dados do estudo de concepção ou da alternativa a ser avaliada. 


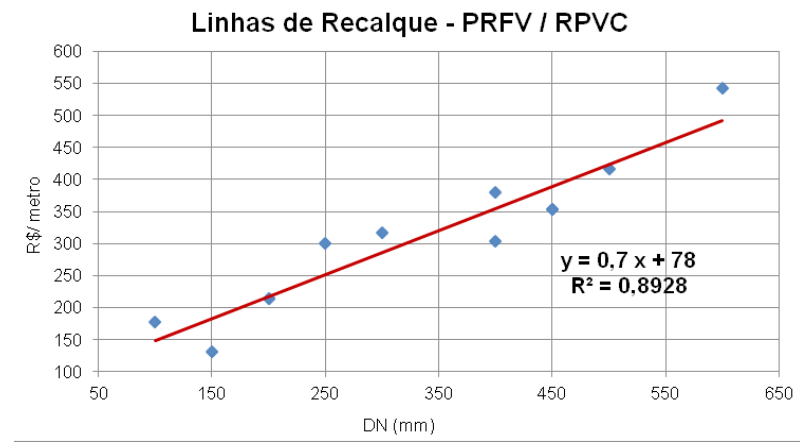

Figura 10 - Custo de Linhas de Recalque - PRFV / RPVC

\section{CONCLUSÕES E RECOMENDAÇÕES}

Foram desenvolvimentos métodos para composições e estimativas de custos, buscando orientar a tomada de decisão, na coleta e afastamento dos esgotos, demonstrados através de tabelas e curvas, para dar subsídio às tomadas de decisões e no auxílio ao desenvolvimento dos Estudos de Concepção.

Quanto às redes coletoras de esgoto, foram pré-dimensionadas e orçadas quatro situações (solo favorável e baixa urbanização - nível 3 e DN $150 \mathrm{~mm}=\mathrm{R} \$$ 137,97/m; solo desfavorável e baixa urbanização - nível 3 e DN150 mm = R\$ 170,53/m; solo favorável e alta urbanização - nível 3 e DN150 $\mathrm{mm}=\mathrm{R} \$ 169,77 / \mathrm{m}$; e solo desfavorável e alta urbanização nível 3 e DN150 mm= R $\$ 210,87 / \mathrm{m})$.

O material orçado foi o PVC (amplamente utilizado) com
DN 150 a 350 mm e diferentes níveis de terreno (1 a 5), como pode ser observado nos resultados (tabela 5 a 8), totalizando 100 resultados de custo de rede coletora de esgoto.

A tabela 9 sumariza os resultados das equações de custos obtidas para Coletores, Interceptores, Estações Elevatórias de Esgoto e Linhas de Recalque.

Os custos apresentados neste artigo possuem base de atualização orçamentária de maio de 2011. Entretanto, utilizandose do fator multiplicador igual a 1,154 (INCC) têm-se os custos atualizados para o primeiro semestre de 2013. Para datas futuras também é possível atualizar, através dos novos índices do INCC.

Recomenda-se como desdobramento deste trabalho, referente aos custos de Sistemas de Esgotamento Sanitário, que sejam incluídos e/ou estudados os custos de manutenção e operação, tais como mão de obra, insumos, energia, entre outros.

Tabela 9 - Resumo dos Resultados e Limites das Equações de Custos

\begin{tabular}{|c|c|c|c|}
\hline Componente & Equação & Limites da Equação & Nota: \\
\hline \multirow[t]{2}{*}{$\begin{array}{l}\text { Coletores e } \\
\text { Interceptores }\end{array}$} & $y=0,0014 x^{2,3}$ & DNs 200 a $400 \mathrm{~mm}$ & $\begin{array}{l}\text { DNs próximos a }-400 \mathrm{~mm} \text { não são } \\
\text { financeiramente viáveis, pois o material } \\
\text { classificado desta equação foi o } \mathrm{PVC}- \\
\text { menos competitivo que o concreto armado } \\
\text { da EQ } 2\end{array}$ \\
\hline & $y=0,0017 x^{2,1}$ & DNs 400 a $800 \mathrm{~mm}$ & ----- \\
\hline \multirow{4}{*}{$\begin{array}{l}\text { Estações Elevatórias } \\
\text { de Esgoto }\end{array}$} & $y=98.000 \ln (x)+250.000$ & $\mathrm{H}_{\operatorname{man}}$ de até 15 m.c.a & Vazões entre 0 a $40 \mathrm{~L} / \mathrm{s}$ \\
\hline & $y=191.000 \ln (x)+50.000$ & $\mathrm{H}_{\operatorname{man}}$ de 15 até 30 m.c.a & Vazões entre 0 a $380 \mathrm{~L} / \mathrm{s}$ \\
\hline & $y=290.000 \ln (x)+140.000$ & $\mathrm{H}_{\operatorname{man}}$ de 30 até 45 m.c.a & Vazões entre 0 a $200 \mathrm{~L} / \mathrm{s}$ \\
\hline & $y=775.000 \ln (x)-1.200 .000$ & $\mathrm{H}_{\operatorname{man}}$ acima de 45 m.c.a & Vazões entre 0 a $260 \mathrm{~L} / \mathrm{s}$ \\
\hline \multirow{4}{*}{ Linhas de Recalque } & $y=4,3 x^{0,8}$ & Material em PEAD & DN 75 à 400 mm \\
\hline & $y=2,6 x^{0,8}$ & $\begin{array}{c}\text { Material em PVC } \\
\text { DeFoFo }\end{array}$ & $\begin{array}{l}\text { DN } 75 \text { à } 300 \mathrm{~mm} \\
\text { Mais econômico até DN } 300 \mathrm{~mm}\end{array}$ \\
\hline & $y=170 \ln (x)-470$ & Material FoFo & DN 80 à 400 mm \\
\hline & $y=0,7 x+78$ & $\begin{array}{c}\text { Materiais PRFV e } \\
\text { RPVC }\end{array}$ & DN 75 à 600 mm \\
\hline
\end{tabular}




\section{REFERÊNCIAS}

ANA - Agência Nacional das Águas. Termos de Referência para Elaboração dos Serviços de Estimativas Orçamentárias para as Intervenções em Captação, Adução e Tratamento de Água e Tratamento de Esgotos. Atlas de Abastecimento de Água do Nordeste, Regiões Metropolitanas e Sul. Brasília: Agência Nacional das Águas, 2008.

BRUDEKI, N.; AISSE, M. M. Custos Estruturais por Habitante em Saneamento Básico no Estado do Paraná. In: CONGRESSO BRASILEIRO DE ENGENHARIA SANITÁRIA E AMBIENTAL, 24., 2007, Belo Horizonte. Anais... [S.l.: s.n.], 2007.

COBRAPE-ENGECORPS-GEOAMBIENTE. Nota técnicametodologia de cálculo das curvas de custo. Brasília: Agência Nacional das Águas, 2009.

COLOSSI, N. Modelos paramétricos para projetos de sistemas de esgotamento sanitário. 2002. Dissertação (Mestrado em Engenharia de Produção) - Universidade Federal de Santa Catarina, Santa Catarina, 2002.

HAGAPLAN-COBRAPE. Resultados dos Dimensionamentos das Redes Coletoras por Sub-Bacias. Estudo de Concepção. Projeto de Engenharia e Projeto Executivo dos Sistemas de Esgotamento Sanitário de Porto Velho. Porto Velho: [s.n.], 2008.

LUCCA, V. P.; SAMWAYS, G.; AISSE, M. M. Estudo dos Custos de Implantação e Operação de Sistemas de Coleta e Tratamento de Esgotos Sanitários para Pequenas Comunidades. In: CONGRESSO BRASILEIRO DE ENGENHARIA SANITÁRIA E AMBIENTAL, 26., 2011, Porto Alegre. Anais... [S.l.: s.n.], 2011.

MINISTÉRIO DAS CIDADES. Diagnóstico de Serviços de Água e Esgoto-SNIS 2012. Brasília: Secretaria Nacional de Saneamento Ambiental, 2014.

PACHECO, R. P. Custos para Implantação de Sistemas de Esgotamento Sanitário. 2011. Dissertação (Mestrado em Engenharia de Recursos Hídricos e Ambiental) - Universidade Federal do Paraná, Curitiba, 2011.

SALAZAR, B. L.; VON SPERLING, M. Desenvolvimento de Funções de Custos de Implantação para Redes Coletoras e Interceptores. In: CONGRESSO BRASILEIRO DE ENGENHARIA SANITÁRIA E AMBIENTAL, 26., 2011, Porto Alegre. Anais... [S.l.: s.n.], 2011.

TSUTIYA, M.; ALÉM SOBRINHO, P. Coleta e Transporte de Esgoto Sanitário. Departamento de Engenharia Hidráulica e Sanitária da Escola Politécnica da Universidade de São Paulo. São Paulo: [s.n.], 1999. 